\title{
Accuracy of ultrasonography in diagnosing acute appendicitis during pregnancy based on surgical findings
}

\author{
Alireza Kazemini ${ }^{1}$, Mohammad Reza Keramati ${ }^{1 *}$, Mohammad Sadegh Fazeli ${ }^{1}$, Amir Keshvari $^{1}$, \\ Siavash $\mathrm{Khaki}^{1}$, Ata Rahnemai-Azar ${ }^{1}$
}

Received: 25 Feb 2017

Published: 29 Aug 2017

\begin{abstract}
Background: Acute appendicitis is the most common nonobstetric surgical problem in pregnancy. Common signs and symptoms of appendicitis are less reliable during pregnancy due to physiological changes; thus, the role of imaging becomes prominent. Thus, in the present study, we aimed at assessing the accuracy of sonography in diagnosing acute appendicitis during pregnancy.

Methods: In this prospective analytic study, among 1000 patients diagnosed and treated as acute appendicitis, clinical and sonographic findings of 58 consecutive pregnant patients, who underwent appendectomy, were recorded and analyzed. All surgically resected samples were evaluated and confirmed through histological evaluation. Sonographic criteria were utilized to judge the results for appendicitis. Diagnostic test performance characteristics (sensitivity, specificity, predictive values, and likelihood ratios) were calculated.

Results: The mean age of the patients was $27.1 \pm 4.9$ years, and the most common clinical symptom was right lower quadrant pain. There was no significant difference in the mean leukocyte count between the appendicitis group and normal appendix group ( $\mathrm{p}=0.768$ ). Left shift was also unrelated with the appendix pathology $(\mathrm{p}=0.549)$. The sensitivity, specificity, predictive values (positive and negative), and likelihood ratios (positive and negative) were $80 \%, 75 \%, 91.4 \%, 52.9 \%, 3.2$, and 0.26 , respectively during all trimesters of pregnancy.

Conclusion: Ultrasonography is the initially preferred imaging modality in pregnant women suspected of having acute appendicitis with an acceptable sensitivity; however, application of other imaging modalities such as CT scan or MRI is recommended after inconclusive ultrasonography results.
\end{abstract}

Keywords: Appendicitis, Sonography, Surgical resection, Predictive value, Likelihood ratio

Copyright@ Iran University of Medical Sciences

Cite this article as: Kazemini A, Keramati MR, Fazeli MS, Keshvari A, Khaki S, Rahnemai-Azar A. Accuracy of ultrasonography in diagnosing acute appendicitis during pregnancy based on surgical findings. Med J Islam Repub Iran. 2017 (29 Aug);31:48. https://doi.org/10.14196/mjiri.31.48

\section{Introduction}

Acute appendicitis is one of the most common surgical acute abdominal diseases (1). The lifetime risk for an appendectomy is $12.0 \%$ in males and $23.1 \%$ in females $(2$, 3 ). The incidence of appendicitis is about 1 in 1700 pregnancies and is almost similar to that of the general population, as gestation does not predispose to appendicitis (4, 5). Acute appendicitis during pregnancy is the most common nonobstetric emergency reported in $25 \%$ of nonobstetric surgical interventions $(6,7)$.

The diagnosis is established by clinical examination and other diagnostic measures. This is a more challenging

Corresponding author: Dr Mohammad Reza Keramati,dr_morezak@yahoo.com

${ }^{1 .}$ Department of Surgery, Tehran University of Medical Sciences, Tehran, Iran. problem during pregnancy because of physiological changes. Accordingly, the rate of perforation is higher than the nonpregnant population, which is the leading cause of mortality and morbidity. Appendicitis increases the rate of spontaneous abortions, preterm delivery, small for gestation babies, and neonatal mortality in the first 7 days of life (4). Moreover, perforated appendicitis is the most common surgical cause of fetal loss in pregnancy $(8$, 9). This makes the diagnosis of appendicitis more important during pregnancy. It has been recognized that early and accurate diagnosis of appendicitis leads to an im-

$\uparrow$ What is "already known" in this topic:

Ultrasonography is the most preferred initial imaging modality in the assessment of suspected cases of appendicitis during pregnancy, but results show a surprisingly high variation between centers and even within a single center.

$\rightarrow$ What this article adds:

Considering the sensitivity and specificity of sonography during pregnancy, other imaging modalities might be needed for the suspected patients. 
proved patient outcome (10). Imaging techniques are useful in the timely diagnosis and clinical assessment of appendicitis (3). Radiation exposure has always been an important concern in managing pregnant patients; and ultrasound is usually the first study attempted.

The present study aimed at assessing the value of sonography in the diagnosis of appendicitis in pregnant patients with high clinical suspicion for appendicitis, who were recommended aggressive surgical exploration, before the development of a complication.

\section{Methods}

This was a prospective single-center study conducted at Imam Khomeini hospital, Tehran, Iran. A total of 58 pregnant women admitted to the emergency department, who were highly suspected of having acute appendicitis and underwent surgical exploration from January 2014 to January 2016, were included in the study. Their medical records were reviewed to collect data on age, weeks of pregnancy, symptoms, signs, and other clinical findings. Results of laboratory tests, reports of ultrasonography, details of the operation, histological findings, and postoperative complications were also noted. Records that did not include pathological assessment or were incomplete were excluded. Those patients treated conservatively were also excluded.

Appendectomies performed during pregnancy were done in collaboration with an obstetrician, and all were open procedures. Simultaneous Cesarean section was done based on the clinical impression of an obstetrician. Histological evaluation was done to approve the diagnosis. Imaging reports were reviewed, and sonographic criterion was used to judge the result positive for appendicitis. Thus, at least one of Puylaert criteria had to be presented, which are as follow: (1) noncompressible, swollen appendix with a diameter greater than $7 \mathrm{~mm}$, and wall thickness greater than 3mm; (2) appendicolith; (3) an increase and hyperechogenicity of periappendiceal fat; (4) lack of normal wall layer; (5) appendiceal abscess; and (6) periappendiceal fluid collection.

Statistical analysis was performed using SPSS 22. Diagnostic test performance characteristics (sensitivity, specificity, positive predictive value, negative predictive value, positive and negative likelihood ratio) were calculated. Statistical significance was set at $p<0.05$.

\section{Results}

Patients

Among 1000 patients diagnosed and treated as acute appendicitis during the study period, clinical and sonographic findings of 58 pregnant patients who underwent appendectomy were recorded. In this study, 63 pregnant patients underwent surgery, while 5 were excluded due to their incomplete medical records. The mean age of patients was $29.1 \pm 4.94$ years. There were $37(63.8 \%), 15$ $(25.9 \%)$, and $6(10.3 \%)$ patients in the first, second, and third trimesters of pregnancy, respectively. The mean gestational age of patients was $13 \pm 8.96$ weeks.

\section{Clinical features}

The most common clinical symptom was right lower quadrant (RLQ) abdominal pain. In this study, 26 patients (44.8\%) had low-grade fever; and the mean \pm SD leukocyte count was $14200 \pm 2580 / \mathrm{mL}$, and leukocyte count was above $16000 / \mathrm{mL}$ in 13 patients. No significant difference was found in the mean leukocyte count between the appendicitis group and normal appendix group ( $\mathrm{p}$-value $=$ 0.768). Left shift was not associated with the appendix pathology $(p$-value $=0.549)($ Table 1$)$.

\section{Sonographic findings}

Ultrasonography was performed for all patients (Tables 2 and 3). The right ovarian cyst was detected in 8 patients, which was concurrent with appendicitis in 2 of the participants. The left ovarian cyst was found in 2 patients and their histological evaluations showed that they both had appendicitis. Sensitivity, specificity, positive predictive value, negative predictive value, positive likelihood ratio, and negative likelihood ratio of sonography for detecting acute appendicitis in the first trimester of pregnancy were $92 \%, 66.7 \%, 92 \%, 66.7 \%, 2.78$, and 0.12 , respectively. The values for the second trimester were $63.7 \%, 75 \%$, $87.5 \%, 42.8 \%, 2.52$, and 0.49 , respectively. The values for the third trimester were $50 \%, 100 \%, 100 \%, 50 \%$, $\mathrm{NA}(\mathrm{x} / 0)$, and 0.5 , respectively.

Moreover, during all trimesters of pregnancy, sensitivity, specificity, positive predictive value, and negative predictive values were $80 \%, 75 \%, 91.4 \%, 52.9 \%$, 3.2 , and 0.26 , respectively.

Table 1. Frequency Distribution of the Clinical Findings of the Patients

\begin{tabular}{llcccc}
\hline Clinical findings & Value & Trimester 1 & Trimester 2 & Trimester 3 & Total \\
\hline Vomiting & Yes & $3(8.1)^{*}$ & $2(13.3)$ & $4(66.7)$ & $9(17.3)$ \\
& No & $34(81.9)$ & $13(87.2)$ & $2(33.3)$ & $49(94.2)$ \\
Pain Location & RLQ (Migration+) & $28(75.7)$ & $3(20)$ & - & $31(59.6)$ \\
& RLQ (Migration-) & $9(24.3)$ & $4(26)$ & - & $13(25)$ \\
& Right umbilical & - & $8(53.3)$ & - & $8(15.3)$ \\
& Right flank & - & - & $6(100)$ & 6 \\
Diarrhea & Yes & $1(2.7)$ & & $1(16.6)$ & $2(3.8)$ \\
& No & $36(97.2)$ & & $5(83.3)$ & $41(78.8)$ \\
Active UA & Yes & $31(83.8)$ & $10(66.7)$ & $6(100)$ & $47(90)$ \\
Leukocyte shift & Yo & $6(16.2)$ & $5(33.3)$ & - & $11(21.15)$ \\
& No & $8(21.6)$ & $6(40)$ & $4(66.7)$ & $18(34.6)$ \\
& *N(\%) & $29(78.4)$ & $9(60)$ & $2(33.3)$ & $40(76.9)$ \\
\hline
\end{tabular}


Table 2. Frequency Distribution of the Sonographic Findings in the Patients

\begin{tabular}{|c|c|c|c|}
\hline Sonographic findings & Trimester 1 & Trimester 2 & Trimester 3 \\
\hline Appendicitis & $25(67.7)^{*}$ & $8(53.3)$ & $2(33.3)$ \\
\hline Not appendicitis & $6(16.2)$ & $7(46.7)$ & - \\
\hline Not done & $6(16.2)$ & - & - \\
\hline Inconclusive /undiagnostic & - & - & $4(66.7)$ \\
\hline Total & $37(100)$ & $15(100)$ & $6(100)$ \\
\hline
\end{tabular}

Table 3. Intraoperative Findings and Simultaneous Operations in the Patients

\begin{tabular}{lcc}
\hline $\begin{array}{l}\text { Intraoperative findings and/or } \\
\text { simultaneous operations }\end{array}$ & Trimester 1 & Trimester 2 \\
\hline Normal appendix & $5(13.5)$ & $2(13.3)$ \\
Acute appendicitis & $23(62.2)$ & $8(53.3)$ \\
Catarrhal appendicitis & $6(16.2)$ & $2(13.3)$ \\
Cystectomy & $1(2.7)$ & $3(50)$ \\
Acute appendicitis +cystectomy & $2(5.4)$ & - \\
Oophorectomy & - & $1(6.7)$ \\
Total & $37(100)$ & $1(6.7)$ \\
\end{tabular}

\section{Operative and histological findings}

Ovarian cystectomy was conducted in all the patients with an ovarian cyst. In one case, oophorectomy was done due to intraoperative situation. The perforation occurred in 2 of the patients $(3.8 \%)$; one of them was in her first trimester, and the other in her third trimester (Table 3). Histological findings after pathologic examination of the samples were compatible with intraoperative findings of the appendix samples. The Initial stages of an inflammatory process in appendix wall without suppuration were reported in early and catarrhal appendicitis. Histologic findings in the supportive forms were transmural inflammatory cell infiltrate, mucosal ulceration, and luminal purulent material. In all trimesters, from a total of 35 patients with preoperative sonographic diagnosis of acute appendicitis, diagnosis was confirmed intraoperatively in only 32 cases. On the other hand, from a total of 17 patients without preoperative sonographic diagnosis, acute appendicitis was detected in 8 patients during the surgical operation.

\section{Postoperational obstetric features}

Uterine contractions were present in 11 patients, and miscarriage occurred in $2(3.8 \%)$ patients, who were in their second trimester. There were $3(5.7 \%)$ appendectomy induced deliveries in the third trimester, one concluded a preterm baby, and 2 underwent simultaneous Cesarean section. Other patients responded to drug therapy. There was no maternal mortality.

\section{Discussion}

In the present study, 58 pregnant patients diagnosed and treated as acute appendicitis during the 3 trimesters of pregnancy were evaluated. To assess the accuracy of sonography in this group of patients, sonographic evaluation was done in all the patients and compared with postoperative findings. Moreover, preoperative clinical examination and intraoperative findings were also recorded in all the patients.

Clinical evaluations are usually the first suggestive clues of acute appendicitis. Typical symptoms of acute appendicitis in nonpregnant patients include diffuse abdominal pain, anorexia, nausea, vomiting, and migration of pain to the right lower quadrant. However, such a classic history might not present in pregnancy. The most common pain location has been found to be in RLQ, but notably every patient in her third trimester had the right flank pain, which was congruent with the relocation of appendix during pregnancy. Meanwhile, laboratory findings were not reported to be helpful in the diagnosis (11, 12). Leukocyte count in pregnancy may range from 6000 to 16000 cells $/ \mathrm{mL}$ and it is still considered a physiological change even with left shift, and thus cannot be relied on as an indicator of appendicitis. During labor, the count may rise as high as 20000 to 30000 cells $/ \mathrm{mL}$, but leukocytosis is not detected in all pregnant patients with appendicitis (13). Higher frequency of abnormal urine analysis in women in their third trimester might be attributed to an ongoing appendix inflammatory process in proximity to the right ureter because of the relocation of the appendix. In the study performed by Tudidor et al. (14) on 500 patients undergoing surgery for acute appendicitis, pyuria ( $>10$ cells per high-power field) was detected in 1/7, and more than 3 red blood cells per high-power field were found in $1 / 6$ of urinary sediments.

The lack of reliability of clinical clues used in nonpregnant patients makes the diagnosis of acute appendicitis even more difficult. Some other signs of appendicitis such as positive Rovsing's and Psoas sign are not important in diagnosing acute appendicitis during pregnancy (11). Anderson et al. (15) evaluated the clinical records of 56 pregnant women undergoing appendectomies and concluded that abdominal pain, nausea, vomiting, leukocyte count, C-reactive protein, and body temperature were not helpful in establishing a correct diagnosis. The mean body temperature in patients with appendicitis was 37.6 degrees C (range: 35.5-39.4) compared with 37.8 degrees C (range: 36.7-38.9) in those with normal histological findings (not significant).

Due to the lack of specificity, preoperative diagnosis, when imaging studies are not used, has been reported to be correct in $1 / 3$ to $3 / 4$ of the cases; accordingly, there is an unacceptable rate of false negative appendectomies $(11,16,17)$, which even increases in second and third trimesters. The need to decrease false negative operations and surgical related mortality and morbidity should be 
balanced with the need for prompt intervention to avoid appendix perforation. Delay in diagnosis increases other problems such as perforation and its resultant complications. Perforation of appendicitis during pregnancy has been reported in $25 \%$ to $40 \%$ of patients (1), compared with $4 \%$ to $19 \%$ reported in the general population (11, 18). Peritonitis is more severe during pregnancy because omentum fails to wall off the appendix in the expanding abdomen due to its limited ability for migration (19). Surgical morbidity and mortality increase sharply when the appendix perforates. Mortality increases from $0.0002 \%$ to $3 \%$, and morbidity increases from $3 \%$ to $47 \%$ (3). Mortality rates have dramatically declined considering the report of Babler in 1908 for the maternal mortality of 24\% and the fetal mortality of $40 \%$ (20). Mortality during appendicitis is due to delay in diagnosis and operation. The risk of fetal loss, resulting from an acute appendicitis during pregnancy is $1.5 \%$ to $10 \%$ if the appendix does not rupture, and it is as high as $36 \%$ if it does (21). A maternal mortality of $0 \%$ to $2 \%$ has been reported $(11,22)$.

Ultrasonography has always been the most preferred initial imaging modality in the assessment of abdominal pain during pregnancy due to lack of ionizing radiation, no need for patient preparation or contrast medium, being rapid, noninvasive, inexpensive, dynamic information through graded compression, and wide availability. However, it is operator- dependent and can be difficult to interpret in cases of obesity, bowel gas, a retrocecal appendix, and severe abdominal pain. Moreover, it may be of particularly limited value in the third trimester because the gravid uterus changes the position of the appendix and prevents the graded compression technique. Being operator- dependent, diagnostic performance results show a surprisingly high variation between centers and even within a single center. A systematic review based on 14 studies reported that ultrasonography had an overall sensitivity of 0.86 , a specificity of 0.81 , a positive likelihood ratio of 5.8, and a negative likelihood ratio of 0.19 to detect acute appendicitis in nonpregnant adults and adolescents (23). In another study on 35 pregnant patients (9), ultrasonography had a sensitivity of $46.1 \%$ and a specificity of $95.4 \%$ to diagnosis appendicitis. Some studies reported ultrasonography to be nondiagnostic in $70 \%$ of the patients during pregnancy (16). Similar to our research, in a recent study on 52 pregnant patients undergoing surgery, Yilmaz et al. (17) reported a sensitivity of $48 \%$ and specificity of $100 \%$, with an accuracy (total correct diagnosis) of 50\% for ultrasonography. In our study, sensitivity, specificity, positive and negative likelihood ratio of sonography were $80 \%, 75 \%, 3.2$, and 0.26 , respectively. The high specificity, but relatively low sensitivity of this study compared with our study showed lower false negative results, but higher false positive results of ultrasonography in our study. This may be due to the fact that the population studied was highly suspected for appendicitis, clinically leading them to surgical intervention in our center. The high number of false negative appendectomies may lead to increased surgical associated mortality and morbidity. To decrease these risks, it is recommended to use another imaging modality such as CT scan or MRI in patients suspected of having appendicitis after normal or inconclusive ultrasonography results $(9,16)$.

Several studies have shown that CT scan is more accurate in the diagnosis of acute appendicitis during pregnancy $(16,24,25)$. Mahesh et al. (9) evaluated the records of 39 pregnant patients suspected of having acute appendicitis referred for imaging and found a sensitivity of $100 \%$ for CT in the diagnosis of appendicitis compared with a sensitivity of $46.1 \%$ for ultrasound. In another study, Wallace et al. (26) retrospectively compared the value of ultrasonography and ultrasonography, followed by CT scan in pregnant patients suspected of having acute appendicitis, who underwent appendectomies. The false negative rate of appendectomy was $36 \%(20 / 55)$ in exclusive ultrasound group and $8 \%(1 / 13)$ in the ultrasound and CT scan group. The prominent concern for CT scanning in pregnancy is a risk of fetal harm from prenatal irradiation (27). The excess relative risk (ERR) of developing childhood cancer is 0.037 at $1.0 \mathrm{mGy}$ during pregnancy, estimating theoretically about one childhood cancer per 500 fetuses, which is twice as general control populations (2). However, interpretation of these figures is still controversial, and the American College of Radiology still recommends to use nonionizing techniques such as ultrasonography and MRI as the first choice in the evaluation of pregnant women $(2,28)$.

MRI is a relatively new technology recently used in the diagnosis of acute appendicitis, with its accuracy reported to be as comparable to CT scan and can be an alternative for pregnant patients $(9,29,30)$. Pedrosa et al. (30) retrospectively assessed the diagnostic importance of MRI in pregnant patients suspected of having acute appendicitis and found a sensitivity and specificity of $100 \%$ and $93.6 \%$, respectively. In a meta-analysis on 229 cases, the sensitivity, specificity, positive and negative predictive values of MRI for diagnosing antenatal appendicitis were $95.0 \%, 99.9 \%, 90.4 \%$, and $99.5 \%$, respectively (31). There are some concerns about the potential harm to fetus due to the effects of magnetic fields and the tissue heating from radiofrequency pulses or acoustic stimulation, however, there are no reported harmful effects $(32,33)$.

\section{Conclusion}

Although sonography is a helpful imaging modality with no ionizing radiation, considering the sensitivity and specificity of this test during pregnancy, other imaging modalities might be needed in suspected patients.

\section{Conflict of Interests}

The authors declare that they have no competing interests.

\section{References}

1. Ueberrueck T, Koch A, Meyer L, Hinkel M, Gastinger I. Ninety-four appendectomies for suspected acute appendicitis during pregnancy. World J Surg. 2004;28(5):508-11.

2. Hurwitz LM, Yoshizumi T, Reiman RE, Goodman PC, Paulson EK, Frush DP, et al. Radiation dose to the fetus from body MDCT during early gestation. AJR Am J Roentgenol. 2006;186(3):871-6.

3. Rybkin AV, Thoeni RF. Current concepts in imaging of appendicitis. Radiol Clin North Am. 2007;45(3):411-22, vii.

4. Flexer S, Tabib N, Peter M. Suspected appendicitis in pregnancy. The 
Surgeon. 2014;12(2):82-6.

5. Wei PL, Keller JJ, Liang HH, Lin HC. Acute appendicitis and adverse pregnancy outcomes: a nationwide population-based study. J Gastrointestinal Surg. 2012;16(6):1204-11.

6. Juhasz-Böss I, Solomayer E, Strik M, Raspé C. Abdominal surgery in pregnancy - an interdisciplinary challenge. Dtsch Arztebl Int. 2014;111:465-72.

7. Neto F, Amorim MMRd, Nóbrega BMSV. Acute appendicitis in pregnancy: literature review. Revista da Associação Médica Brasileira. 2015;61(2):170-7.

8. Parangi S, Levine D, Henry A, Isakovich N, Pories S. Surgical gastrointestinal disorders during pregnancy. Am J Surg. 2007; 193(2):223-32.

9. Shetty MK, Garrett NM, Carpenter WS, Shah YP, Roberts C. Abdominal computed tomography during pregnancy for suspected appendicitis: a 5-year experience at a maternity hospital. Semin Ultrasound CT MR. 2010;31(1):8-13.

10. Bouyou J, Gaujoux S, Marcellin L, Leconte M, Goffinet F, Chapron $\mathrm{C}$, et al. Abdominal emergencies during pregnancy. J Visceral Surg. 2015;152(6):S105-S15.

11. Pastore PA, Loomis DM, Sauret J. Appendicitis in pregnancy. J Am Board Fam Med. 2006;19(6):621-6.

12. Maslovitz S, Gutman G, Lessing JB, Kupferminc MJ, Gamzu R. The significance of clinical signs and blood indices for the diagnosis of appendicitis during pregnancy. Gynecol Obstet Invest. 2003;56(4):188-91.

13. Sharp HT. Gastrointestinal surgical conditions during pregnancy. Clin Obstet Gynecol. 1994;37(2):306-15.

14. Tundidor Bermudez AM, Amado Dieguez JA, Montes de Oca Mastrapa JL. [Urological manifestations of acute appendicitis]. Arch Esp Urol. 2005;58(3):207-12.

15. Andersen B, Nielsen TF. Appendicitis in pregnancy: diagnosis, management and complications. Acta Obstet Gynecol Scand. 1999;78(9):758-62.

16. Basaran A, Basaran M. Diagnosis of acute appendicitis during pregnancy: a systematic review. Obstet Gynecol Surv. 2009; 64(7):481-8; quiz 99.

17. Yilmaz HG, Akgun Y, Bac B, Celik Y. Acute appendicitis in pregnancy-risk factors associated with principal outcomes: a case control study. Int J Surg. 2007;5(3):192-7.

18. Tracey M, Fletcher HS. Appendicitis in pregnancy. Am Surg. 2000;66(6):555-9; discussion 9-60.

19. Epstein FB. Acute abdominal pain in pregnancy. Emerg Med Clin North Am. 1994;12(1):151-65.

20. Gomez A, Wood M. Acute appendicitis during pregnancy. Am J Surg. 1979;137(2):180-3

21. Al-Fozan H, Tulandi T. Safety and risks of laparoscopy in pregnancy. Curr Opin Obstet Gynecol. 2002;14(4):375-9.

22. Ghazanfar A, Nasir SM, Choudary ZA, Ahmad W. Acute appendicitis complicating pregnancy; experience with the management of 50 patients. J Ayub Med Coll Abbottabad. 2002; 14(3):19-21.

23. Terasawa T, Blackmore CC, Bent S, Kohlwes RJ. Systematic review: computed tomography and ultrasonography to detect acute appendicitis in adults and adolescents. Ann Intern Med. 2004; 141(7):537-46.

24. Lazarus E, Mayo-Smith WW, Mainiero MB, Spencer PK. CT in the evaluation of nontraumatic abdominal pain in pregnant women. Radiology. 2007;244(3):784-90.

25. Ames Castro M, Shipp TD, Castro EE, Ouzounian J, Rao P. The use of helical computed tomography in pregnancy for the diagnosis of acute appendicitis. Am J Obstet Gynecol. 2001;184(5):954-7.

26. Wallace CA, Petrov MS, Soybel DI, Ferzoco SJ, Ashley SW, Tavakkolizadeh A. Influence of imaging on the negative appendectomy rate in pregnancy. J Gastrointest Surg. 2008;12(1):4650.

27. Kal HB, Struikmans H. [Pregnancy and medical irradiation; summary and conclusions from the International Commission on Radiological Protection, Publication 84]. Ned Tijdschr Geneeskd. 2002;146(7):299-303.

28. Ralls PW, Balfe DM, Bree RL, DiSantis DJ, Glick SN, Levine MS, et al. Evaluation of acute right lower quadrant pain. American College of Radiology. ACR Appropriateness Criteria. Radiology. 2000;215 Suppl:159-66.

29. Cobben LP, Groot I, Haans L, Blickman JG, Puylaert J. MRI for clinically suspected appendicitis during pregnancy. AJR Am J Roentgenol. 2004;183(3):671-5.

30. Pedrosa I, Levine D, Eyvazzadeh AD, Siewert B, Ngo L, Rofsky NM. MR imaging evaluation of acute appendicitis in pregnancy. Radiology. 2006;238(3):891-9.

31. Blumenfeld YJ, Wong AE, Jafari A, Barth RA, El-Sayed YY. MR imaging in cases of antenatal suspected appendicitis - a meta-analysis. J Matern Fetal Neonatal Med. 2010.

32. Duncan KR. The development of magnetic resonance imaging in obstetrics. Br J Hosp Med. 1996;55(4):178-81.

33. Kirkinen P, Partanen K, Vainio P, Ryynanen M. MRI in obstetrics: a supplementary method for ultrasonography. Ann Med. 1996; 28(2):131-6. 